\title{
Too Little of a Good Thing: Hydroxychloroquine in Pregnancy
}

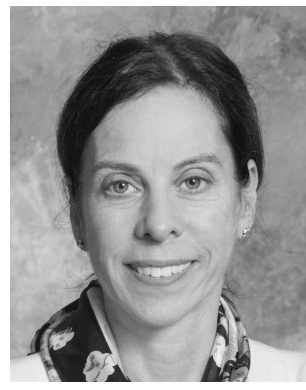

Women with rheumatic diseases, including inflammatory arthritis and systemic lupus erythematosus (SLE), fare better in pregnancy when their disease is under good control ${ }^{1,2}$. The role of hydroxychloroquine (HCQ) for achieving this control is now recognized. Several studies demonstrate that patients with SLE who continue HCQ during pregnancy have decreased flares and improved pregnancy outcomes, including longer fetal gestation and infants with higher birth weight ${ }^{3,4}$. Reassuringly, antimalarials are compatible with pregnancy, with no signals for safety concerns, and professional society guidelines recommend continuation of HCQ during pregnancy ${ }^{5}$. In support of this approach, a survey of North American rheumatologists found that over 69\% of rheumatologists continued HCQ in their pregnant patients ${ }^{6}$.

Despite the recognition that HCQ is a key component of SLE disease management in both nonpregnant and pregnant patients, adherence to HCQ remains abysmally low. Feldman, et al used claims data to show that $79 \%$ of nonpregnant patients with SLE are nonadherent ${ }^{7}$. Although claims data have also showed that overall use of HCQ during pregnancy has improved from $12.4 \%$ in 2004 to $37.7 \%$ in 2015 , these rates are far from optimal ${ }^{8}$. Thus, low adherence to HCQ confounds conclusions regarding this drug's effect on disease control and pregnancy outcome in SLE.

To circumvent the possible effect of medication nonadherence on the understanding of the role of HCQ in rheumatic disease management, literature has focused on the measurement of either whole blood or serum drug levels and correlating these levels to disease activity. Costedoat-Chalumeau, et al were among the first to show that HCQ concentration could predict disease activity, with whole blood drug levels of $>1000 \mathrm{ng} / \mathrm{ml}$ having a high negative predictive value of flare ${ }^{9}$. Mok, et al measured HCQ levels in sera and defined 3 groups of patients based on drug level: those with drug levels $<10 \mathrm{ng} / \mathrm{ml}$ were classified as noncompliant; those with levels of $10-500 \mathrm{ng} / \mathrm{ml}$ were classified as having subtherapeutic drug levels; and a third group with levels $>500 \mathrm{ng} / \mathrm{ml}$ were classified as having therapeutic levels. This study was unable to establish a statistically significant relationship between serum level of HCQ and disease activity. It concluded that drug levels themselves cannot differentiate noncompliance from other pharmacokinetic factors ${ }^{10}$.

HCQ drug level during pregnancy is likely to be even more unpredictable given the increased volume of distribution of the drug and the increase in the body mass index of pregnant women. Nonetheless, the evaluation of HCQ level during pregnancy could provide valuable information for disease management. In this issue of The Journal, Balevic, et al report on a single-center observational study of 50 pregnant patients with rheumatic disease who were prescribed $\mathrm{HCQ}^{11}$. The authors measured serum levels of HCQ in all 3 trimesters and related these levels to disease activity and pregnancy outcomes. HCQ levels were categorized as nontherapeutic $(<100 \mathrm{ng} / \mathrm{ml})$ or therapeutic $(>100 \mathrm{ng} / \mathrm{ml}$ ). The latter category was further divided into suboptimal therapeutic levels, defined as drug level of 100 $\mathrm{ng} / \mathrm{ml}-500 \mathrm{ng} / \mathrm{ml}$, and optimal therapeutic levels, defined as drug level $>500 \mathrm{ng} / \mathrm{ml}$. Twenty-eight patients had SLE, 7 had rheumatoid arthritis or juvenile idiopathic arthritis, 5 had connective tissue disease, and the remaining 10 had other rheumatic and autoimmune disorders. In patients with SLE, disease activity was assessed by physician's global assessment (PGA); SLE Disease Activity Index (SLEDAI) scores; and C3, C4, and dsDNA levels. High disease activity for patients with SLE was defined as a PGA > 1.0. In the non-SLE patients, disease activity was measured by PGA on a visual analog scale ranging from $0 \mathrm{~mm}$ (no disease activity) to $100 \mathrm{~mm}$ (highest disease activity), with high disease activity defined as $>25 \mathrm{~mm}$. Primary outcome for this study was neonatal gestational age. Preterm birth (defined as $<37$ weeks) and birth weight were secondary outcomes.

The authors found significant intrasubject variability in serum HCQ during pregnancy ${ }^{11}$. Twenty-four percent of patients had at least $1 \mathrm{HCQ}$ level $<100 \mathrm{ng} / \mathrm{ml}$, suggesting a high rate of nonadherence. Among the SLE pregnancies, those with nontherapeutic levels of HCQ $(<100 \mathrm{ng} / \mathrm{ml})$ had a higher frequency of infants with lower gestational age $(p=0.03)$; however, the authors were unable to demonstrate a linear association between HCQ serum level and gestational age. The authors also found a higher frequency of infants preterm born to mothers with a serum level of HCQ

See HCQ levels during pregnancy, page 57

Personal non-commercial use only. The Journal of Rheumatology Copyright (c) 2019. All rights reserved. 
$<100 \mathrm{ng} / \mathrm{ml}$ compared to those with therapeutic levels of HCQ $(>100 \mathrm{ng} / \mathrm{ml} ; \mathrm{p}=0.01)^{11}$. Nonetheless, the rate of preterm births among those patients with optimal therapeutic levels of HCQ (>500 ng/ml) was $80 \%$, nearly identical to that in the nontherapeutic group (83\%). Surprisingly, mean birth weight was the lowest in the group with optimal therapeutic levels of HCQ. These paradoxical findings could reflect that the patients in the $>500 \mathrm{ng} / \mathrm{ml}$ group may have been sicker. Eighty-three percent of patients in this group compared to $12 \%$ in the suboptimal therapeutic group and $16.7 \%$ in the nontherapeutic groups were taking azathioprine (AZA), suggesting that these patients may have had disease that is more complex. Alternatively, AZA itself could contribute to preterm and lower birth weights ${ }^{12}$.

Although the authors found a statistically significant relationship between HCQ levels and disease activity as measured by the PGA, the $\mathrm{r}^{2}$ of 0.07 reflects a nonlinear relationship. One possible explanation for this poor correlation is that the mean PGA among the patients with SLE was $<1.0$ in all $3 \mathrm{HCQ}$ therapeutic ranges, reflecting overall low disease activity in this cohort. This low disease activity may have diluted the authors' findings - HCQ level may have a larger effect in those patients with more active disease.

In patients with SLE, neither the SLEDAI nor serum markers of SLE disease activity correlated with HCQ levels. In the non-SLE patient group, neither disease activity nor pregnancy outcome was related to HCQ levels; however, the numbers of subjects in each diagnostic subcategory were so small that no definitive conclusions could be drawn.

The authors of the aforementioned study should be lauded for their efforts to obtain serum levels of HCQ in 3 trimesters of pregnancy, demonstrating a proof of concept that this is a feasible approach to SLE pregnancy management ${ }^{11}$. The finding that nontherapeutic levels of HCQ were associated with a higher frequency of lower gestational age and more preterm delivery supports the authors' conclusion that targeting strategies to improve HCQ adherence in pregnancy may improve outcome, although the lack of linear correlation between HCQ level and gestational age suggests that this relationship is complex. The paradox that high serum levels > $500 \mathrm{ng} / \mathrm{ml}$ were likewise associated with both poor pregnancy outcome and higher disease activity remains confusing.

The relative merits of whole blood versus serum measurement of HCQ are unknown. As the authors point out, while whole blood testing may be more precise, serum levels may have the advantage of being less prone to confounding by cytopenias ${ }^{11}$. Comparing these approaches head to head may clarify this point. The optimal duration of therapy to achieve steady state, timing of blood draw in relation to dose administration, changes in pharmacokinetics because of pregnancy, and disease factors still represent unresolved issues. Further studies into the pharmacokinetics of HCQ in the pregnant and nonpregnant state will also be important.

This study is a good start in understanding the relationship between HCQ levels, disease activity, and pregnancy outcome ${ }^{11}$, and suggests that maybe we ought to be focusing on drug levels rather than the dose of HCQ in our patients with SLE, whether pregnant or not. Importantly, whatever metric is used to determine HCQ adherence, many of our patients with SLE remain undertreated, and this could affect both disease activity and pregnancy outcome.

BONNIE L. BERMAS, MD

UT Southwestern Medical Center, Division of Rheumatic Diseases, Dallas, Texas, USA.

Address correspondence to Dr. B.L. Bermas, UT Southwestern Medical Center, Rheumatic Diseases, 2001 Inwood Road, Dallas, Texas 75390, USA.E-mail: Bonnie.Bermas@UTSouthwestern.edu

\section{REFERENCES}

1. Skorpen CG, Lydersen S, Gilboe IM, Skomsvoll JF, Salvesen KA, Palm Ø, et al. Influence of disease activity and medications on offspring birth weight, pre-eclampsia and preterm birth in systemic lupus erythematosus: a population based study. Ann Rheum Dis 2018;77:264-9

2. Chakravarty EF. Rheumatoid arthritis and pregnancy: beyond smaller and preterm babies. Arthritis Rheum 2011;63:1469-71.

3. Eudy AM, Siega-Riz AM, Engel SM, Franceschini N, Howard AG, Clowse MEB, et al. Effect of pregnancy on disease flares in patients with systemic lupus erythematosus. Ann Rheum Dis 2018;77:855-60.

4. Liu EL, Liu Z, Zhou YX. Feasibility of hydroxychloroquine adjuvant therapy in pregnant women with systemic lupus erythematosus. Biomed Res 2018;29:980-3.

5. Flint J, Panchal S, Hurrell A, van de Venne M, Gayed M, Schreiber $\mathrm{K}$, et al. BSR and BHPR guideline on prescribing drugs in pregnancy and breastfeeding-Part I: standard and biologic disease modifying anti-rheumatic drugs and corticosteroids. Rheumatology 2016;55:1693-7.

6. Al-Herz A, Schulzer M, Esdaile JM. Survey of antimalarial use in lupus pregnancy and lactation. J Rheumatol 2002;29:700-6.

7. Feldman CH, Yazdany J, Guan H, Solomon DH, Costenbader KH. Medication nonadherence is associated with increased subsequent acute care utilization among Medicaid beneficiaries with systemic lupus erythematosus. Arthritis Care Res 2015;67:1712-21.

8. Bermas BL, Kim SC, Huybrechts K, Mogun H, Hernandez-Diaz S, Bateman BT, et al. Trends in use of hydroxychloroquine during pregnancy in systemic lupus erythematosus patients from 2001-2015. Lupus 2018 Jan 1; E-pub ahead of print.

9. Costedoat-Chalumeau N, Amoura Z, Hulot JS, Hammoud HA, Aymard G, Cacoub P, et al. Low blood concentration of hydroxychloroquine is a marker for and predictor of disease exacerbations in patients with systemic lupus erythematosus. Arthritis Rheum 2006;54:3284-90.

10. Mok CC, Penn HJ, Chan KL, Tse SM, Langman LJ, Jannetto PJ. Hydroxychloroquine serum concentrations and flares of systemic lupus erythematosus: A longitudinal cohort analysis. Arthritis Care Res 2016;68:1295-302.

11. Balevic SJ, Cohen-Wolkowiez M, Eudy AM, Green TP, Schanberg LE, Clowse MEB. Hydroxychloroquine levels throughout pregnancies complicated by rheumatic disease: implications for maternal and neonatal outcomes. J Rheumatol 2019;46:57-63.

12. Pirson Y, Van Lierde M, Ghysen J, Squifflet JP, Alexandre GP, van Ypersele de Strihou C. Retardation of fetal growth in patients receiving immunosuppressive therapy. N Engl J Med 1985;313:328.

J Rheumatol 2019;46:1-2; doi:10.3899/jrheum.181046

Personal non-commercial use only. The Journal of Rheumatology Copyright (c) 2019. All rights reserved. 